\title{
XLVIII. The maintenance of forced oscillations of a new type
}

\section{C.V. Raman M.A.}

To cite this article: C.V. Raman M.A. (1912) XLVIII. The maintenance of forced oscillations of a new type, Philosophical Magazine Series 6, 24:142, 513-520, DOI: 10.1080/14786441008637356

To link to this article: http://dx.doi.org/10.1080/14786441008637356

册 Published online: 08 Jun 2010.

Submit your article to this journal $\sqsubset \pi$

Џll Article views: 5

Q View related articles $₫$ 
For soda glass, the kind used by Professor More in his electrostriction experiments, we find

$\begin{array}{cc}c . & e j e^{\prime} . \\ +1.06 & 0.03 \\ -1.06 & 0.11 \\ 0 & 0.07\end{array}$

Thus the elongation to be looked for is certainly less than 11 per cent. of that which Prof. More expected, and his conclusion that the elongations he obtained were caused by heating is confirmed by these results.

With the Jena glass tube considerably greater elongations can be looked for, as shown by the following table :-

$\begin{array}{cc}c . & e / e^{\prime} . \\ +0 \cdot 62 & 0 \cdot 22 \\ -0.62 & 0 \cdot 28 \\ 0 & 0.25\end{array}$

It is just possible that using glass tubes of this kind a true elongation caused by electrostriction might he observed.

In conclusion, it appears that one will not go far wrong in taking for solid dielectrics, as for fluid dielectrics, $\delta_{1}=\delta_{2}$. Assuming this, the calculation of $\delta_{2}-\sigma\left(\delta_{1}+\delta_{2}\right)$ given in the previous paper from Wüllner and Wien's experiments gives at once the values of $\delta_{1}=\delta_{2}$ for the kinds of glass which they used.

Palmer Physical Laboratory, Princeton, New Jersey.

XLVIII. 'The Maintenance of Forced Oscillations of a New Type. By C. V. Raman, M.A.*

[Plates X.-XII.]

THE photographs published with this paper (Pls. X.-XII.) represent some of the very remarkable class of maintained oscillations regarding which preliminary communications were published in the issues of 'Nature' dated the 9th Dec., 1909, and the 10th Feb., 1910, under the title of the present paper. I propose here to confine myself to indicating the main features of the phenomena actually observed in experiment, and to elucidating these by the aid of a physical theory that I have since worked out.

One of the most fascinating problems in the dynamics of

* Communicated by the Author. 
vibrating bodies is the behaviour of a system when subject to periodic forces which tend merely to alter its "spring" or restitutional coefficient, and do not directly tend to displace the system from its position of equilibrium. In discussing this problem, it is assumed that this periodic change in "spring" is of definite frequency and is imposed from outside the system. One principal point for inquiry is the relation that must exist between this frequency and that of the free oscillations of the system in order that the latter may be permanently maintained in vibration in the presence of dissipative forces. Lord Rayleigh has investigated the particular case in which the frequency of the imposed force is double that of the oscillations of the system, and has shown that under suitable circumstances the oscillation may be maintained. The question arises whether there do not exist other frequency-ratios which would permit of the maintenance of the oscillations of the system. Mr. Andrew Stephenson attacks this problem by pushing to a higher degree of approximation the analytical method employed by Hill in discussing certain problems in the Lunar Theory, and by Lord Rayleigh in working out the particular case of double frequency. His analysis (published in the Quarterly Journal of Pure and Applied Mathematics for June 1906) leads to the result that the oscillations of the system may be magnified or maintained under suitable circumstances, if the frequency $\mathrm{N}$ of the imposed variation of spring stands to the frequency $\mathrm{N}_{1}$ of the oscillation in the relation $2: r$ where $r$ is any positive integer. When $r$ is unity, we evidently have the case of double frequency referred to above.

From an acoustical point of view, the possibility of maintenance is that which possesses the greatest interest. Mr. Stephenson's treatment is purely mathematical, and his paper does not explain in terms of ordinary physical ideas why it is that the oscillations should admit of being maintained with the specified frequency-relations, nor does he offer any experimental evidence in support of such a proposition. It is necessary therefore to discuss the problem in its physical bearings before any results of experiment can be rendered intelligible.

If the oscillations of the system are to be maintained in the presence of dissipative forces, a continued supply of energy from without into the system is clearly necessary, and we have therefore to show that with the assumed relation between the frequency of the imposed variation of spring and that of the oscillations of the system, it is possible for a finite amount of energy to pass into the system during every 
complete period of the variation of the spring, and that this is just sufficient to balance the loss by dissipation during the same time. This again would only be possible, if, assuming that an oscillation of any particular frequency was maintained, the product of the imposed variation of spring into the displacement, which may be regarded as the impressed part of the force tending to restore the system to its position of equilibrium, had a periodic component of the same frequency as that of the oscillation proposed to be maintained, and this should also be of a suitable magnitude and phase. This is evident from the general principles of resonance, since components of the restoring force having any other frequency would obviously be comparatively ineffective in maintaining the vibration.

The condition referred to above, it may readily be shown, is satisfied in the case of double frequency. Thus, putting $\mathrm{N}$ and $\mathrm{N}_{1}$ equal to $n / 2 \pi$ and $n_{1} / 2 \pi$ respectively, and assuming that the variation of spring is represented by $-2 a n_{1}{ }^{2} \sin n t$ and the displacement $\phi$ at any instant is equal to $\mathrm{C} \cos \left(n t / 2+\epsilon_{1}\right)$, it is seen that the product of the variation of spring and the displacement has a periodic component $-\alpha \mathrm{C} n_{1}{ }^{2} \sin \left(n t / 2-\epsilon_{1}\right)$ which has the same frequency as the oscillation proposed to be maintained. It is rendily shown that the work done by this part of the force acting on the system in a time equal to the period of the variation of spring is $\alpha / 4 n_{1}{ }^{2} n \mathrm{C}^{2} t \cos 2 \epsilon$, and that this is equal to the loss by dissipation in the same time if $\kappa n=\alpha n_{1} \cos 2 \epsilon_{1}, 2 \kappa n_{1}$ being the dissipation coefficient. This result, it will be seen, is precisely equivalent to that obtained in a different manner by Lord Rayleigh (Scientific Works, vol. ii. p. 192).

Taking now the general case discussed by Stephenson, we may first assume that a motion of frequency $r \mathrm{~N} / 2$ is maintained by the variation of spring $-2 \alpha n_{1}^{2} \sin n t$. If $\phi$ the displacement at any instant is put equal to $\mathrm{C}_{r} \cos \left(r n t / 2+\epsilon_{r}\right)$, where $\mathrm{C}_{r}$ is a constant, it is readily seen that the product of the variation of spring and the displacement which may be termed the impressed part of the restoring force is $-2 \alpha_{r} n_{2}^{2} \sin n t \cos \left(r n t / 2+\epsilon_{r}\right)$, and this has obviously no component of frequency $r \mathrm{~N} / 2$ and cannot therefore maintain an oscillation having that frequency. The expression for the work done by it in a time equal to the period of the variable spring will be found equal to zero. The inference is that, if the oscillation is actually maintained, some important term in the expression for the displacement at any instant bas been omitted by us to be taken into account. This we now proceed to find directly from physical considerations. 
We may, to fix our ideas, consider a case in which the frequency of the oscillations of the system is large compared with the frequency of the imposed variation of spring. It is clear in this case that the successive oscillations of the system are not executed under identical conditions. At one epoch the restitutional coefficient is a maximum, and an oscillation would evidently be executed by the system during this epoch in a shorter time than at the epoch when the spring is a minimum, and the amplitude of the oscillation would also be greater at the latter epoch. The motion of the system in such a case would evidently be similar to that in the atmospheric beats produced by two simple tones one of which has a greater frequency and amplitude than the other (Appendix XIV. of Helmholtz's 'Sensations of Tone'). We have therefore to modify the expression for the displacement applicable in the general case and may assume it to be approximately represented by two terms, one of which has a much larger amplitude than the other and has a frequency in excess of it by that of the imposed variation of spring.

We may therefore put $\phi$ the displacement equal to

$$
\mathrm{C}_{r} \cos \left(r n t / 2+\epsilon_{r}\right)+\mathrm{C}_{r-2} \cos \left(\overline{r-2} n t / 2+\epsilon_{r-2}\right) .
$$

The expression for the restoring force at any instant requires corresponding modification. The product of the variation of spring and the displacement is

$$
-2 \alpha n_{1}{ }^{2} \sin n t\left[\mathrm{C}_{r} \cos \left(r n t / 2+\epsilon_{r}\right)+\mathrm{C}_{r-2} \cos \left(\overline{r-2} n t / 2+\epsilon_{r-2}\right)\right] \text {. }
$$

It is readily seen that this has a periodic component of frequency $r \mathrm{~N} / 2$ which is equal to

$$
-\alpha n_{1}^{2} \mathrm{C}_{r-2} \sin \left(r n t / 2+\epsilon r_{-2}\right) \text { if } r>2 \text {, }
$$

or

$$
-2 \alpha n_{1}^{2} \mathrm{C}_{r-2} \sin n t \cos \epsilon_{r-2} \text { if } r=2 \text {. }
$$

The work done by this the "impressed" part of the restoring force during a period of the variation of spring is readily shown to be

$$
\begin{gathered}
-\alpha / 4 r n_{1}^{2} n \mathrm{C}_{r} \mathrm{C}_{r-2} t \cos \left(\epsilon_{r}-\epsilon_{r-2}\right), \text { if } r>2 \\
\text { or } \quad-\alpha n_{1}^{2} n \mathrm{C}_{r} \mathrm{C}_{r-2} t \cos \epsilon_{r} \cos \epsilon_{r-2}, \text { if } r=2 .
\end{gathered}
$$

The energy dissipated during the sume time is

$$
\frac{1}{4} \kappa n^{2} n_{1}\left[r^{2} \mathrm{C}_{r}^{2}+(r-2)^{2} \mathrm{C}_{r-2}^{2}\right] t \text {. }
$$

Since the 2nd term within the square brackets is the square of a small quantity it may be neglected, and the energy supplied is equal to the energy dissipated if

provided $r>2$, or if

$$
\kappa m \mathrm{C}_{r}=-\alpha n_{1} \mathrm{U}_{r-2} \cos \left(\epsilon_{r}-\epsilon_{r-2}\right)
$$

$$
\kappa r n \mathrm{C}_{r}=-2 \alpha n_{1} \mathrm{C}_{r_{-2}} \cos \epsilon_{r} \cos \epsilon_{-2} \text { when } r=2 .
$$


It is thus seen that the 2nd term in the expression for the displacement is from a physical point of view of very great importance, though its amplitude may be small compared with that of the 1st term which is much the larger part of the oscillation actually maintained. It is not developed or brought out in the final expression for the magnified or maintained motion as given by Stephenson, and the importance of the part played by it in magnifying or maintaining the motion is therefore not made evident in his paper.

It seems well to consider a few numerical examples. If the frequency of the imposed variation of "spring" were 60 per second, the oscillations of the system would be maintained (under suitable circumstances) if the frequency of its free oscillations were nearly equal to 30 or 60 or 90 or $1 \% 0$ or 150 and so on, the degree of approximation to equality necessary increasing as we proceed up the series. The frequency of the maintained oscillation would be exactly 30 or 60 or 90 and so on. But in the case of the oscillation of frequency 60 , the motion would be very approximately represented by a periodic term of that frequency plus a small constant. In the case of the 3rd type in the series, the maintained oscillation would be represented by a periodic term of frequency 90 plus a small term of frequency 30. In the 4th case, the motion would be represented by a periodic term of frequency 120 plus a small term of frequency 60 and so on, the 2nd term in each type being less in frequency than the 1st term by 60 , which is the frequency of the variation of spring; in the case of the 2 nd type in which the frequency of the first term is itself 60 , the 2 nd term naturally assumes the form of a constant as stated above. The significance of this is that an oscillation when maintained by a variation of spring of the same frequency has for its mean point, not the equilibrium position of the system, but one slightly displaced to one side thereof. This result is no doubt somewhat paradoxical, but there is nothing absurd in it, inasmuch as we are here dealing with motion under variable spring in the presence of dissipative forces. The restoring forces at the points of maximum displacement on either side of the equilibrium position may be equal in mannitude and opposite in sign despite the fact that these displacements from the equilibrium position are themselves slightly difforent, and a steady oscillation about a displaced mean point is therefore possible. It is readily seen that in this case it is the slight displacement of the mean point of the normal oscillation that enables a surplus of energy to Phil. Mag. S. 6. Vol. 24. No. 142. Oct. 1912. $2 \mathrm{M}$ 
pass from without into the system to counteract the effect of dissipation and thrus maintain the motion.

Thy class of maintained vibrations discussed above can be realized in experiment and caused to yield some very beautiful results that will now be described. The vibrating systems used in the work are stretched strings which seem prima facie well adapted for acoustical work on this subject. Important advantages are that their vibrations are visible without any aid, and, secondly, that their frequency can be adjusted with ease and accuracy over a comparatively wide range. With stretcher strings again, it is comparatively an easy matter to impose a perindic variation of "spring", i.e. of tension in this case, of any desired magnitudo and frequency. The experimental arrangements required are fairly simple. A fine string one or two metres in length is taken and one end of it is passed over a peg or a pulley, preferably the former. The other end is attached to the prong of a massive tuning-fork of frequency say 60 or 100 per second, in such a manner that the tension of the string is varied by the motion of the prong. The tuning-fork should be maintained electrically, but for some purposes powerful bowing is sufficient.

It is generally supposed that under these circumstances the equilibrium position of the string becomes unstable and it is thrown into a state of permanent and vigorous vibration only when the frequency of the free oscillations of the string (in its fundamental or other mode) is approximately half of that of the point of attachment. I found that this was not the case. A permanent and vigorous vibration was also maintained when the frequency of the free oscillations of the string was equal to, or one and a half times, or twice, or two and a half times, or thrice that of the fork. This is precisely what is to be expected from the theory dealt with above, and there is absolutely no difficulty in obtaining any one of the first five or six of these types of maiutained oscillation each separately by itself, i.e. with the frequency of the oscillation of the string having the specified relation to that of the tuning-fork. All that is required is that the tension should be so adjusted till the frequency of the free oscillations of the string in its fundamental mode bears the desired relation to the frequency of the fork. This can be done by trial, and it will then generally be found that the oscillations are maintained.

To obtain the results shown in the photographs(Pls.X.-XII.) a somewhat different procedure is adopted. 'The vibrating system here dealt with, i.e. a stretched string, has not merely one free period of oscillation but a series of such free periods 
in which it divides up into one, two, or more segments. Since the frequencies of oscillation which a variable spring of given frequency may maintain under suitable circumstances also form a series, it is evidently possible for more than one mode of vibration to be maintained at one and the same time, each with its own appropriate frequency. In other words, the variable spring may maintain a compound vibration, and as the components of this motion need not both or all be in one and the same principal plane of vibration of the string, we may readily obtain by a little calculation and trial types of maintained motion in which the oscillation in one principal plane is of one frequency and in the perpendicular plane of a different frequency. Under these circumstances, the motion of a point on the string in a plane transverse to it is the appropriate Lissajous figure, and the frequency relation between the component motions is thus rendered evident to inspection in a most striking manner.

The photographs represent short sections of the string thus maintained in stationary vibration, one point in the middle of the section being brilliantly illuminated. Fig. 1 shows the ordinary Ist type of maintenanee in which the frequency of the motion is half of that of the fork. Fig. 2 shows a compound of the 1st and 2nd types in suitable phase relation, the motion being in a parabolic arc. Fig. 3 is a compound of the 1st and 3rd types. Fig. 4 is a compound of the 2 nd and 3rd types which have frequencies respectively equal to and half as much again as that of the fork. Figs. 5 and 6 are complementary, i.e. relate to the same type of oscillation, fig. 5 showing one part of the string and fig. 6 another part. In these two photographs, the 1st and 3rd types of maintained motion occur together in one principal plane, and the 2nd type by itself in the perpendicular plane. In fig. 5 the 1st and 3rd types are in similar phases, but in tig. 6 they are opposed, hence the very remarkable split-ring effect in the latter. In fig. 7 we have the 1st and 3rd types again in perpendicular planes, but along with the 3rd type there is a clear addition of the 2nd type as well. Figs. 8 and 9 are complementary, and show the 1st type maintained in one plane, and the 2nd and 4th types together in the perpendicular plane. Fig. 10 shows a compound of the 2nd and 5th types having frequencies respectively equal to and two and a half times that of the fork. Fig. 11 shows the 1st type in one plane and the 2 nd and 5 th types together in a perpendicular plane. Figs. 12 and 13 are complementary, i. e. show different parts $2 \mathrm{M} 2$ 
of a string under the same conditions. They represent the 1st and 5 th types together in one plane and the $2 \mathrm{nd}$ by itself in the perpendicular plane. In fig. 12, the 1 st and 5 th types are in the same phase, and in fig. 13 they are opposed. Figs. 14 and 15 show the 1st type in one plane, and the 2nd and 6th types together in the perpendicular plane. The two latter are in different relative phases in the two photographs.

Regarding the plate as a whole, it is not a little surprising to contemplate that a strictly normal motion imparted to one extremity of a stretched string should result (under suitable circumstances) in such an extraordinary variety of types of oscillation! I do not think I have photographed one-half of the different types that can easily be maintained permanently.

Conclusion :-I have indicated some of the principal results of experimental interest obtained with this class of maintained vibrations. Further experimental work on the leading types and a detailed comparison with the results of the physical theory which I have outlined above and with the mathematical analysis, does not fall within the limits of the present paper, and I hope to deal with them on an early date. Mean while I must remark that the existence and physical importance of the small "second" term of lower frequency in the expression for the maintained motion is directly demonstrable by strohoscopic observation of each of the five or six types of maintained motion referred to above and by a study of their "vibration-curves." The results of this work are being prepared for publication. The experiments and observations recorded in this note were made at the Physical Laboratory of the Indian Association for the Cultivation of Science, Calcutta.

XLIX. On the Relative Intensities of the Earth's Penetrating Radiation on Land and Sea. By Professor J. C. Molennan, University of Toronto *

\section{Introduction.}

QINCE the discovery of the existence of a penetrating $D$ radiation at the surface of the earth in $1902 t$, numerous measurements of its intensity have been made in different

- Communicated by the Author. Read before the Royal Society of Canada, May 17, 1912.

+ McLeunan \& Burton, Phil. Mag. v. 1903; Cooke, Phil. Mag. vi. 1903. 
Raman.

Fig. 1.

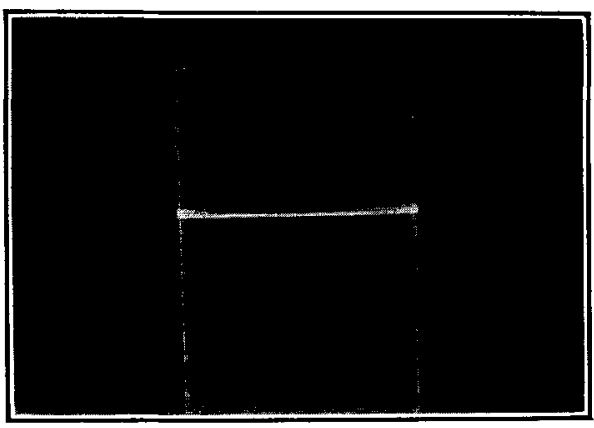

Fig. 3.

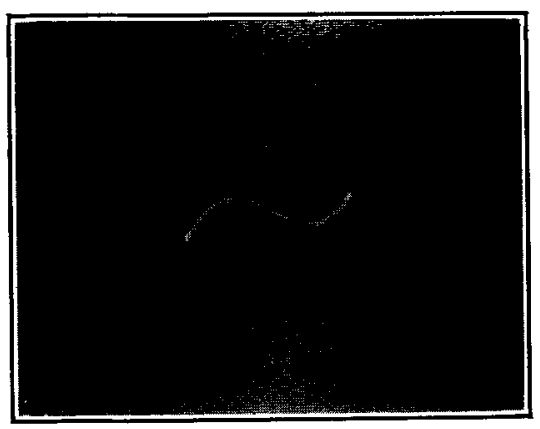

FIG. 5.

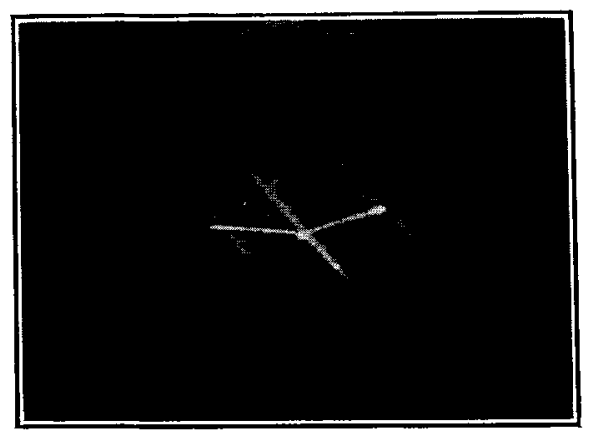

Phil. Mag. Ser. 6, Vol. 24, Pl. X.

FIG. 2.

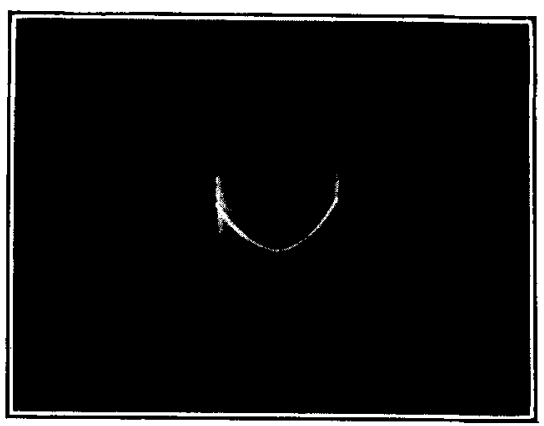

Fig. 4.

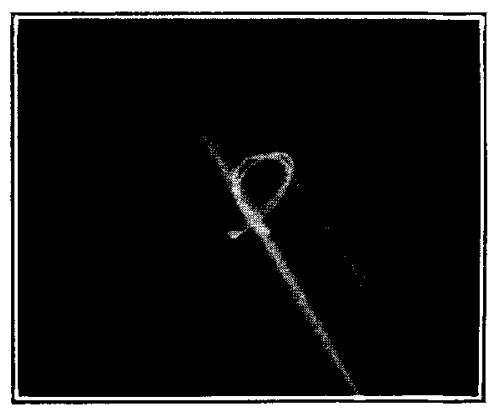

FIG. 6.

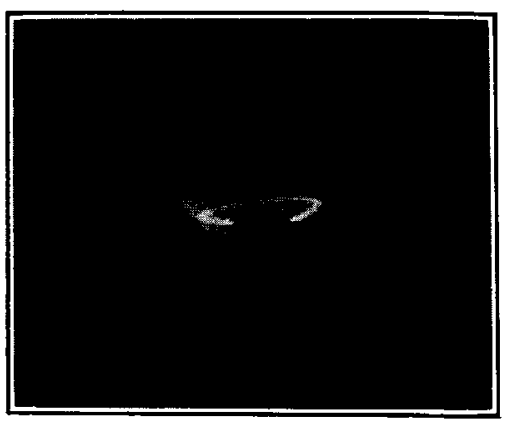


RAMAN.

Fig. 7.

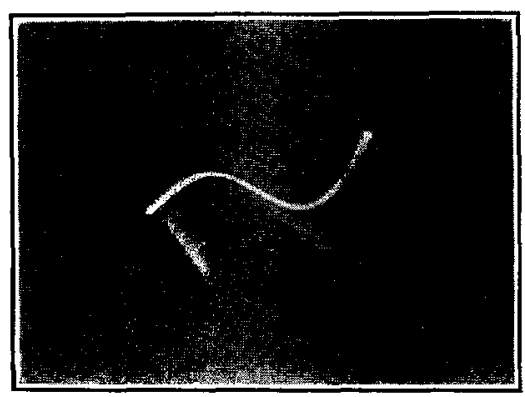

Fig. 9.

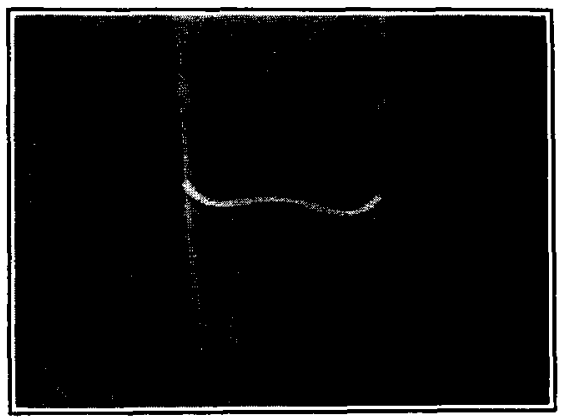

Fia. 11.

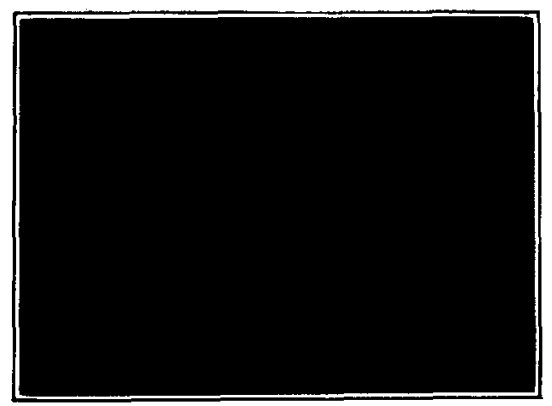

Phil. Mag. Ser. 6, Vol. 24, Pl. XI.

F゙IG. 8.

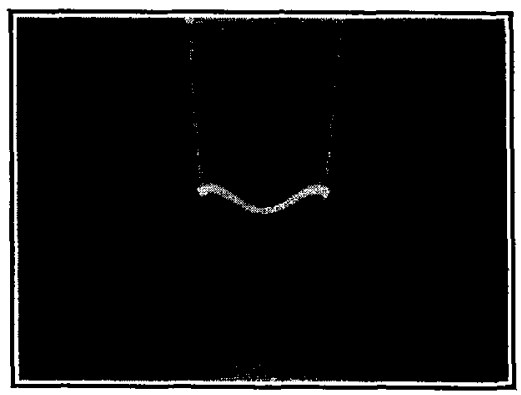

FIG. 10.

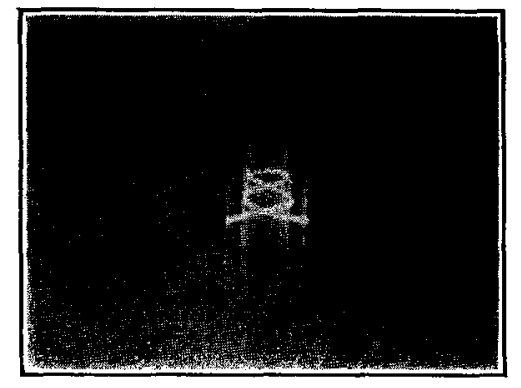

FIG. 12.

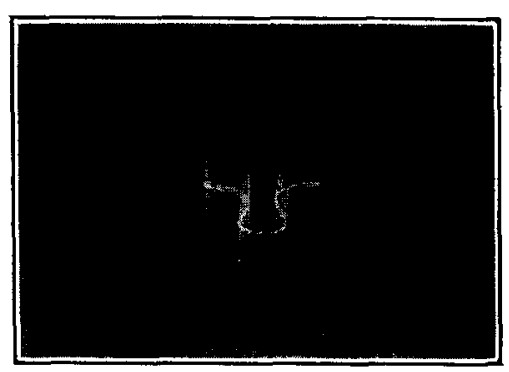


Raman.

Phil. Mag. Ser. 6, Vol. 24, Pl. XII.

FIe. 13.

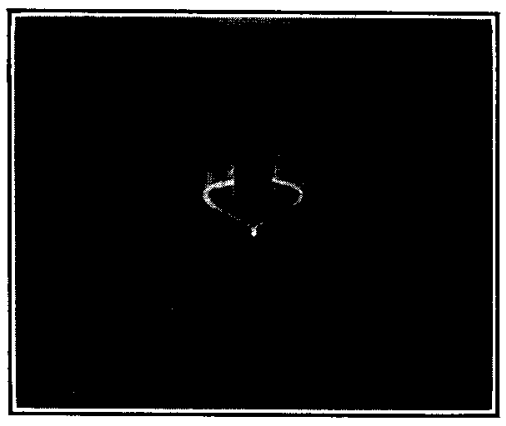

FIG. 14.

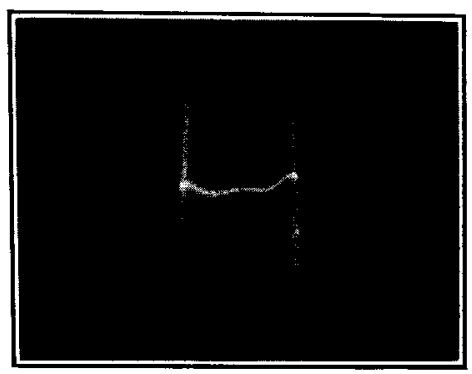

FIG. 15.

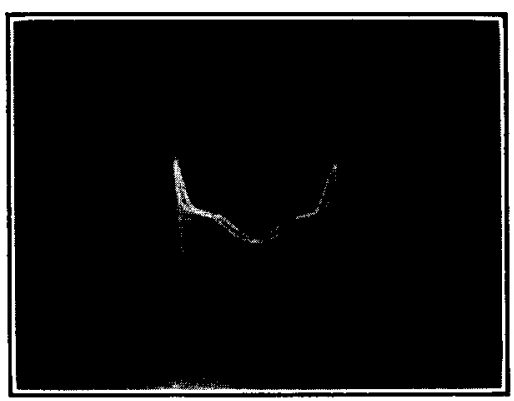

